\title{
Effect of mesenchymal stem cell transplantation on brain-derived neurotrophic factor expression in rats with Tourette syndrome
}

\author{
XIUMEI LIU* ${ }^{*}$ XUEMING WANG ${ }^{*}$, AIMIN LI and XIAOLING JIAO \\ Department of Pediatrics, Yuhuangding Hospital of Qingdao University, Yantai, Shandong 264000, P.R. China
}

Received October 21, 2014; Accepted October 14, 2015

DOI: 10.3892/etm.2016.3059

\begin{abstract}
The aim of the present study was to investigate the effect of bone marrow mesenchymal stem cell (MSC) transplantation on brain-derived neurotrophic factor (BDNF) expression in the striatum of Tourette syndrome (TS) rats. In addition, the possible mechanism of MSC transplantation in the treatment of TS was investigated. A total of 72 Wistar rats were randomly allocated into the control (sham surgery) group and the two experimental groups, including the TS+vehicle and TS+MSC. MSCs were co-cultured with 5-bromodeoxyuridine for $24 \mathrm{~h}$ for labeling prior to grafting. An autoimmune TS rat model was successfully established in the present study. Rat MSCs were cultured and expanded using density gradient centrifugation in vitro, identified by flow cytometry and then transplanted into the striata of the TS+MSC group rats. The mRNA and protein expression levels of BDNF were detected by RT-qPCR and ELISA, respectively. The results indicated that the stereotypic behavior of TS rats was reduced 7 days after MSC transplantation, while the mRNA and protein BDNF levels in the striatum increased, compared with the sham surgery group $(\mathrm{P}<0.05)$. In addition, the BDNF mRNA and protein expression level was lower in the striatum of TS+MSC transplantation, compared with that in TS+vehicle rats. In conclusion, intrastriatal transplantation of MSCs may provide relief from stereotypic TS behavior, since the BDNF level was reduced in TS rats after MSC transplantation.
\end{abstract}

\section{Introduction}

Tourette syndrome (TS) is a chronic neurobehavioral disorder with an unclear etiology and pathophysiology. The primary symptoms of TS are vocal and motor tics, which may result

Correspondence to: Dr Aimin Li, Department of Pediatrics, Yuhuangding Hospital of Qingdao University, 20 Yuhuangding East Road, Yantai, Shandong 264000, P.R. China

E-mail: sdytyhdek@163.com

*Contributed equally

Key words: bone marrow mesenchymal stem cells, transplantation, brain-derived neurotrophic factor, Tourette syndrome in lifelong impairment in certain individuals. In recent years, the prevalence of TS has been increasing (1), and 5\% of TS patients present life-threatening symptoms, which are defined as malignant TS (2). These symptoms are difficult to manage with conservative treatments and neurosurgical procedures.

Recent studies have demonstrated that stem cell-based therapy may be a potential treatment for numerous neurological disorders $(3,4)$. In 2008 , our group transplanted neural stem cells (NSCs) into TS rats and the therapeutic effects of NSCs on the stereotypic behavior of TS rats was investigated (5). However, compared with NSCs, mesenchymal stem cells (MSCs) are a better option for cell transplantation therapy, since they are immunologically inert and easily accessible. In addition, MSCs are able to rapidly expand in cell culture and have been shown to present long-term survival and integration with the host tissue. In a further study in 2010, our group infused MSCs into the striatum of TS rats, revealing that a fraction of MSCs differentiated into neurons and gliocytes (6). Therefore, replacement of neuronal cells by MSCs was hypothesized to contribute to the functional improvement of TS rats. However, the differentiation rate of MSCs observed in our previous study was lower than expected (6). Therefore, assessing the underlying mechanism through which MSCs act to alleviate the symptoms of TS was difficult.

MSCs have also been found to improve the impaired microenvironment induced by central nervous system disease, and to regulate neurotransmitters and neurotrophic factors (7). Our study in 2013 reported that MSC transplantation suppressed the dopamine system and decreased the dopamine levels in the striatum of TS rats (8).

Neurotrophic factors involve in the endogenous protective process of brain injury. Brain-derived neurotrophic factor (BDNF) is one of the most important members of the neurotrophin family and is able to mediate the neuronal growth and differentiation, synapse formation and plasticity, and higher cognitive functions (9). In the present study, the effect of MSC transplantation on the BDNF levels in TS rats was investigated and the possible underlying mechanisms involved in the MSC transplantation were assessed.

\section{Materials and methods}

Animals. A total of 72 Wistar rats (age, 7 weeks; weight, 205-220 g), obtained from Vital River Laboratory Animal Technology Co., Ltd. (Beijing, China), were acclimatized for 
1 week prior to the initiation of the experiments. The animals were housed in a controlled environment, at a room temperature of $21 \pm 1^{\circ} \mathrm{C}$ and a 12 -h light/dark cycle (lights on between 7:00 and 19:00), and had free access to food and water. All experimental procedures were performed in accordance with the NIH Guide for the Care and Use of Laboratory Animals.

MSC preparation and flow cytometric analysis. The long bones of an additional 6 adult Wistar rats (supplied by Shandong University) were used to isolate mononuclear cells, using density gradient centrifugation. In brief, the rat MSCs were isolated by ficoll density gradient centrifugation at $902 \mathrm{x} \mathrm{g}$ for $20 \mathrm{~min}$. The mononuclear cells, located in the middle layer (1-2 mm thickness), were removed by the pipette and centrifuged twice in phosphate-buffered saline (PBS; Sigma-Aldrich, St. Louis, MO, USA) solution at 157 x g for $5 \mathrm{~min}$. Then, mononuclear cells were further cultured with media supplemented with FBS (Gibco; Thermo Fisher Scientific, Inc., Grand Island, NY, USA). Initially, the bones were removed by dissection, and their distal and proximal ends were removed to reveal the marrow cavity. The obtained bone marrow MSCs were cultured in low-glucose Dulbecco's modified Eagle's medium (Gibco; Thermo Fisher Scientific, Inc.) supplemented with $15 \% \mathrm{FBS}, 100 \mathrm{U} / \mathrm{ml}$ penicillin, and $100 \mathrm{mg} / \mathrm{ml}$ streptomycin (Gibco; Thermo Fisher Scientific, Inc.). Next, the cells were incubated at $37^{\circ} \mathrm{C}$ in $5 \% \mathrm{CO}_{2}$ and fresh culture medium was added every 3-4 days. Upon reaching $80 \%$ confluence, trypsin (Sigma-Aldrich) was used to harvest the adherent cells, which were then passaged. Flow cytometry was used to assess $3 \times 10^{7} \mathrm{MSCs}$ at the third passage (FACSCalibur; BD Biosciences, USA). The remaining MSC samples $\left(1 \times 10^{6}\right.$ cells) were co-incubated with medium and $10 \mathrm{mg} / \mathrm{ml}$ bromodeoxyuridine (BrdU; Sigma-Aldrich), a thymidine analog and marker of newly synthesized DNA, for $24 \mathrm{~h}$ prior to transplantation, in order to label the MSCs. The cells were then harvested, resuspended in PBS at a density of $1 \times 10^{5}$ cells $/ \mu 1$ and stored on ice until required for grafting.

Serum of TS patients. A previous study revealed that a high concentration of antibasal ganglia antibody (ABGA) is present in the sera of TS patients, which may result in impairments of the striatum, as well as stereotypic behavior (10). In the present study, serum samples from 8 TS patients (male, 4; female, 4; age range, 8-13 years; mean age, 10.3 years) were obtained from the serum bank of the Yuhuangding Hospital of Qingdao University (Yantai, China). At the time of blood collection, none of the subjects were taking psychostimulants. Serum samples were collected according to a protocol approved by the Institutional Review Board and subsequent to obtaining written informed consent from all the patients. Enzyme-linked immunosorbent assay (ELISA) was performed, as previously described (11), to determine the optical density of the ABGA in the TS patients selected for microinfusion, which was found to be $0.23 \pm 0.07 \mathrm{U} / 1$. The patient serum was obtained and injected into rats in order to increase their ABGA concentration and establish a TS model.

Animal preparation and in vivo surgery. In the present study, an autoimmune TS rat model was established, as previously described (10). The 72 Wistar rats were randomly allocated to the control (sham surgery group, microinfused with normal sera; $n=24)$ and two experimental groups, including the TS+vehicle and TS+MSC groups ( $\mathrm{n}=24$ each). MSCs were co-cultured with BrdU for $24 \mathrm{~h}$ for labeling prior to grafting. Normal serum normal blood samples were obtained from the serum library.

Rats were deeply anesthetized with chloral hydrate (400 mg/kg, intraperitoneal injection) and placed in a stereotaxic apparatus (Stoelting Co., Wood Dale, IL, USA), with the incisor bar set at $3.5 \mathrm{~mm}$ below the interaural line. Through a surgical aseptic technique, the skull was exposed and holes were drilled in appropriate locations, following which a 28-gauge guide cannula was implanted into the bilateral striata. The cannula was placed at the following coordinates: At $2.0 \mathrm{~mm}$ anterior-posterior from bregma, $4.0 \mathrm{~mm}$ medial-lateral and $-7.0 \mathrm{~mm}$ dorsoventral from the skull. The rats were provided with appropriate postsurgical care, with a diet supplemented with egg yolk and fresh fruit, in order to maintain their body weight.

The animals were allowed to recover for 1 week to reestablish the integrity of the blood-brain barrier. Following recovery, Alzet osmotic mini-pumps (Durect Corporation, Palo Alto, CA, USA) filled with PBS were connected to each cannula using a polyethylene tube that was loaded with $50 \mu 1$ undiluted TS or control serum, under sterile conditions. The serum was infused into the rats at a rate of $0.5 \mu \mathrm{l} / \mathrm{h}$ for $72 \mathrm{~h}$, after which the rats were sedated with chloral hydrate $(100 \mathrm{mg} / \mathrm{kg}$, intraperitoneal injection) and placed in the stereotaxic apparatus. The skull was exposed through an incision along the midline and the osmotic mini-pump was removed. In the TS+MSC group, $5 \mu \mathrm{l} /$ site MSC suspension $\left(10^{5}\right.$ cells $\left./ \mu \mathrm{l}\right)$ was bilaterally injected into the sera-infusion sites, one on each side of the rat striatum. After $5 \mathrm{~min}$, the needle was slowly removed and a surgical suture was used to close the wound. Each grafted animal received a total of $10^{6} \mathrm{MSCs}$, with $5 \times 10^{5} \mathrm{MSCs}$ injected into each side of the striatum. For sham grafting, rats in the TS+vehicle group were subjected to the same grafting procedure, but received a vehicle infusion of an equal volume of PBS, rather than MSC suspension. Subsequently, the TS rats were intramuscularly administered 65,000 units of sodium penicillin (Hebei Chengshengtang Animal Pharmaceutical Co., Ltd., Hebei, China) and were maintained on a thermal pad until awakened, after which they were returned to their cages. The animals underwent behavioral assessment tests and were then sacrificed for BDNF detection at 1, 7, 14 and 28 days after transplantation, with 6 rats from each group sacrificed at the different time points.

Assessment of stereotypy. Following the completion of the light cycle, audio and video recordings of the rat movements were obtained for $30 \mathrm{~min}$. Stereotypies were recorded based on previously reported instructions $(12,13)$, and these included head shaking, self-gnawing, biting (which was identified by wood chips, teeth touching the cage, vacuous chewing or biting other objects excluding the rats' body), episodic utterances, grooming, paw shaking, taffy pulling (forepaw to the mouth and face), licking and rearing. Grooming behavior was recorded according to the number of minutes for which grooming occurred. Episodic utterance was determined as repeated medium-pitched sounds of short duration. The 
aforementioned stereotypic movements were recorded at 1, 7, 14 and 28 days after transplantation and a total score was determined for each rat based on the sum of the observed stereotypic movements. The recordings of the rats were reviewed and quantified by a researcher who was trained to identify the aforementioned stereotypies and was blinded to the graft and serum microinfusion details of the rats.

$B D F N$ detection using reverse transcription-quantitative polymerase chain reaction $(R T-q P C R)$. RT-qPCR was performed to determine the mRNA expression of BDNF in the striatum tissues isolated from the rats at 1, 7, 14 and 28 days after transplantation. Total RNA was extracted from the striatum according to the manufacturer instructions of the TRIzol kit (Guangzhou Dongsheng Biotech Co., Ltd., Guangzhou, China). Subsequently, total RNA was reverse transcribed into cDNA in a total reaction volume of $20 \mu \mathrm{l}$, using PrimeScript ${ }^{\mathrm{TM}}$ RT reagent Kit (RR037A; Takara Bio, Inc., Otsu, Japan). The annealed mixture had a volume of $15.5 \mu \mathrm{l}$, including $2 \mu \mathrm{g}$ RNA, $1 \mu \mathrm{l} 0.5 \mathrm{~g} / 1$ oligo dT and $1 \mu \mathrm{l}$ dNTPs, whereas the RT reaction solution was comprised of $2 \mu \mathrm{l} 10 \mathrm{X}$ RT buffer, $1 \mu \mathrm{l}$ dithiothreitol, $0.5 \mu 1$ RNA inhibitors and $1 \mu \mathrm{l}$ M-MLV reverse transcriptase. The $\mathrm{RT}$ reaction was performed in a $37^{\circ} \mathrm{C}$ water bath for $60 \mathrm{~min}$, and then incubated at $70^{\circ} \mathrm{C}$ for $15 \mathrm{~min}$.

Next, qPCR was performed in a total volume of $20 \mu \mathrm{l}$, comprising $2 \mu \mathrm{l}$ DNA template, $10 \mu \mathrm{l}$ Platinum SYBR Green qPCR SuperMix (2X; Thermo Fisher Scientific, Inc., Carlsbad, CA, USA), $0.4 \mu \mathrm{l}$ downstream PCR primers $(10 \mu \mathrm{M}), 0.4 \mu \mathrm{l}$ ROX Reference Dye (50X; Thermo Fisher Scientific, Inc.) and $6.8 \mu \mathrm{l}$ double-distilled $\mathrm{H}_{2} \mathrm{O}$. The primers used in qPCR were designed by Beijing Zhongshan Golden Bridge Biotechnology Co., Ltd. (Beijing, China) according to the GenBank sequences (MIM113505), as follows: BDNF (466 bp), 5'-TCCCTGGCT GACACTTTTGAG-3' (sense) and 5'-ATTGGGTAGTTCGGC ATTGCG-3' (antisense); $\beta$-actin (336 bp), 5'-GCAGAAGGA GATCACAGCCCT-3' (sense) and 5'-GCTGATCCACATCTG CTGGAA-3' (antisense). The reaction system was divided into a 96-well optical plate and covered with a special optical film (Thermo Fisher Scientific, Inc.). Applied Biosystems 7500 Real-Time PCR system (Thermo Fisher Scientific, Inc.) was used for qPCR cycling, as follows: $50^{\circ} \mathrm{C}$ for $2 \mathrm{~min}, 95^{\circ} \mathrm{C}$ for $2 \mathrm{~min}$, then 45 cycles of $95^{\circ} \mathrm{C}$ for $15 \mathrm{sec}$ and $60^{\circ} \mathrm{C}$ for $34 \mathrm{sec}$, followed by $95^{\circ} \mathrm{C}$ for $15 \mathrm{sec}, 60^{\circ} \mathrm{C}$ for $1 \mathrm{~min}$, and $95^{\circ} \mathrm{C}$ for $15 \mathrm{sec}$. The mRNA expression levels were quantified using ABI Prism 7000 software (Thermo Fisher Scientific, Inc.).

BDNF detection using sandwich ELISA. BDNF ELISA kits were used to measure the BDNF protein expression (RAB0026; Sigma-Aldrich). All procedures were conducted according to standard guidelines provided by the manufacturer. The plates included the following samples: i) Blank wells, including a biotin-labeled anti-BDNF antibody and streptavidin-biotin-horseradish peroxidase (HRP); ii) standard wells, including $50 \mu \mathrm{l}$ standard and $50 \mu \mathrm{l}$ streptavidin-biotin-HRP; iii) sample wells, including $40 \mu 1$ sample, followed by addition of $10 \mu \mathrm{l}$ anti-BDNF antibody and $50 \mu 1$ streptavidin-biotin-HRP. The plates were covered using a closure plate membrane, gently shaken, incubated at $37^{\circ} \mathrm{C}$ for $60 \mathrm{~min}$ and then washed. Next, the plates were carefully uncovered to discard the liquid, and then dried and

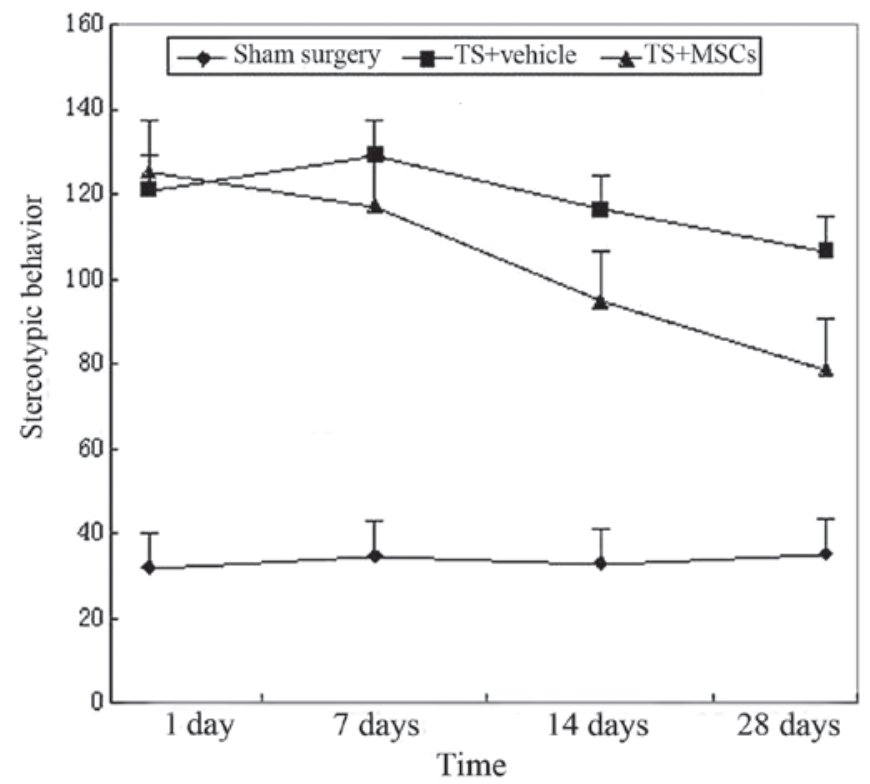

Figure 1. Stereotypic behavior counts were recorded for $30 \mathrm{~min}$ at different time points in the three groups. Scores are higher in the TS groups compared with those in the sham surgery group ( $\mathrm{P}<0.05$ vs. sham surgery rats). In addition, TS+MSCs rats exhibited decreased stereotypic behavior compared with the TS+vehicle rats $(\mathrm{P}<0.05$ vs. TS+vehicle rats). BDNF, brain-derived neurotrophic factor; TS, Tourette syndrome; MSC, mesenchymal stem cell. Figure adapted from Liu et al (8).

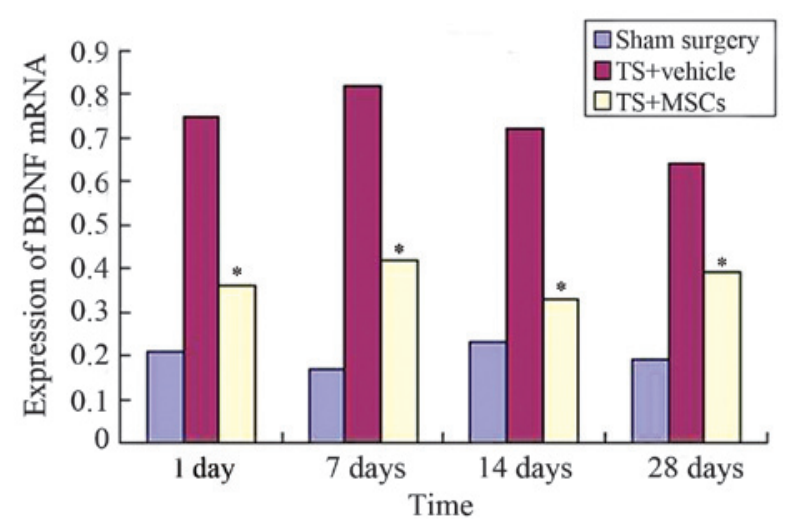

Figure 2. Expression of BDNF mRNA in rat striatum, determined by reverse transcription-quantitative polymerase chain reaction. BDNF mRNA expression reduced in the rat striatum following MSC transplantation (TS+MSCs group), compared with that in the TS+vehicle group ( ${ }^{*} \mathrm{P}<0.05$ vs. TS+vehicle rats). BDNF, brain-derived neurotrophic factor; TS, Tourette syndrome; MSC, mesenchymal stem cell.

washed. The aforementioned procedure was repeated 5 times and then the plates were dried with blotting paper to remove any unbound enzyme-labeled antibody. Subsequently, $50 \mu 1$ chromogenic reagent $\mathrm{A}$ was added to each well, followed by $50 \mu \mathrm{l}$ color reagent $\mathrm{B}$, and the plate were gently mixed, $37^{\circ} \mathrm{C}$ color for $10 \mathrm{~min}$. To terminate the reaction, $50 \mu 1$ stop solution was added to terminate the reaction. Reagents A and B and the stop solution were included in the ELISA kit (Sigma-Aldrich). After $10 \mathrm{~min}$, the absorbance of each well in terms of the optical density (OD) was measured at $450 \mathrm{~nm}$, setting the OD of the blank well to zero. Each plate's concentration was corrected by means of its standard curve's dilution factor. A CCL-2600C microplate reader (Guangzhou Cancare Medical 


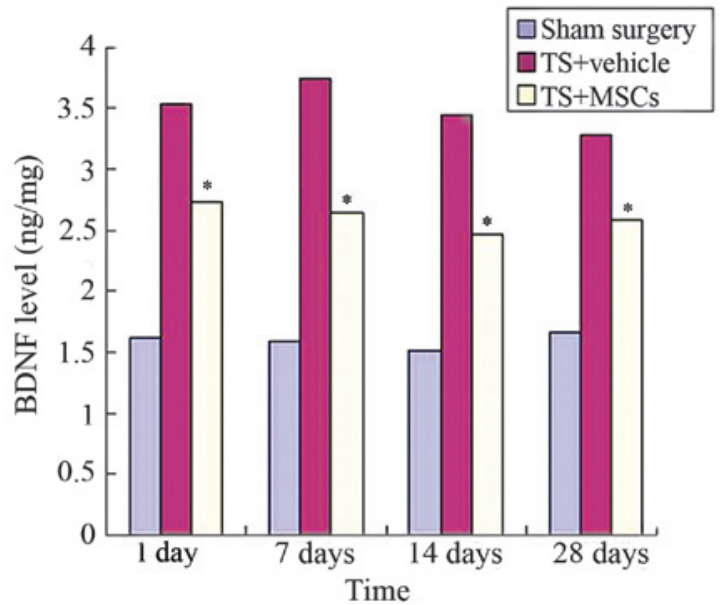

Figure 3. BDNF protein levels in the striata of the TS rat group were higher compared with the sham surgery group $\left({ }^{*} \mathrm{P}<0.05\right.$ vs. sham surgery rats), whereas the levels were lower in the TS+MSC group compared with the TS+vehicle group (" $\mathrm{P}<0.05$ vs. TS+vehicle rats). BDNF, brain-derived neurotrophic factor; TS, Tourette syndrome; MSC, mesenchymal stem cell.

Trading Co., Ltd., Guangzhou, China) was used to determine BDNF absorbance values at $450 \mathrm{~nm}$. The standard linear regression equation was calculated based on the concentration and corresponding OD values, and then the sample BDNF concentration was calculated from the regression equation.

Statistical analysis. All statistical analyses were performed using SPSS (version 13.0 for Windows; SPSS Inc, Chicago, IL, USA). Data are reported as the mean \pm standard deviation. Statistical analysis was performed by repeated measurement analysis of variance in order to evaluate the stereotypy counts at different time points. A P-value of $<0.05$ was considered to indicate a statistically significant difference.

\section{Results}

Assessment of stereotypy. The results indicated that stereotypic TS behavior was successfully established in the rats following intrastriatal microinfusion of serum from TS patients. Stereotypic TS behavior was recorded and quantified in the rats during a 30-min observation period at 1, 7, 14 and 28 days after transplantation (Fig. 1). Statistical analysis indicated that rats in the two TS groups exhibited significantly higher stereotypic behavioral counts compared with those in the sham surgery group $(\mathrm{P}<0.05)$. In addition, TS rats with MSC grafts exhibited significantly decreased stereotypic behavior at 1 week after transplantation $(\mathrm{P}<0.05$; Fig. 1).

Effects of MSC transplantation on the TS rat striatum neurotrophic factor. The mRNA expression levels in the rat striatum were determined using RT-qPCR. Compared with the sham surgery group, the mRNA expression levels of BDNF in the striatum of two TS rat groups showed high levels $(\mathrm{P}<0.05)$. However, BDNF mRNA expression was found to be reduced in the rat striatum following MSC transplantation (TS+MSCs group), compared with that in the TS+vehicle group (Fig. 2).

ELISA was also used to determine the BDNF protein expression in the striatum of TS rats. At 28 days after transplantation, BDNF levels were found to be significantly increased in the TS+MSC group $(3.29 \pm 0.58 \mathrm{ng} / \mathrm{g})$ compared with those in the sham surgery group $(1.67 \pm 0.31 \mathrm{ng} / \mathrm{g} ; \mathrm{P}<0.05)$. In addition, the TS+MSC rats had significantly lower BDNF levels compared with the TS+vehicle rats $(2.59 \pm 0.52 \mathrm{ng} / \mathrm{g}$ vs. $3.29 \pm 0.58 \mathrm{ng} / \mathrm{g}$, respectively; $\mathrm{P}<0.05$ ) (Fig. 3).

Therefore, the findings of the present study demonstrated that transplantation of MSCs was able to reduce the levels of BDNF in the TS rats and alleviate the symptoms of TS.

\section{Discussion}

Current therapeutic approaches for the treatment of TS include psychological and behavioral therapy, drug administration, immunomodulatory therapy and neurosurgery (14). Patients with malignant TS experience self-injurious and life-threatening symptoms, which can not be managed by neurosurgical procedures (such as deep brain stimulation) or conservative treatments. Effective novel strategies for the treatment of TS patients must be developed; thus, the present study investigated an MSC transplantation procedure that was performed into the striatum of a rat TS model. The results demonstrated that transplantation of MSCs resulted in significant improvement of stereotypic behavior in the TS rats (5).

The underlying mechanisms of the MSC transplantation action in the treatment of central nervous system disorders may be as follows (15): i) MSCs may directionally migrate to the injury site and differentiated into Nestin-positive cells, replacing any damaged or dead nerve cells; ii) MSCs may regulate neurotrophic factors, including nerve growth factor and BDNF, which can regulate neuronal survival, mediate axonal growth and regulate neurotransmitter generation; and iii) MSCs may function via immunomodulation.

In recent years, the role of neurotrophic factors in the pathogenesis and treatment of neuropsychiatric disorders was emphasized (16). A neurotrophic factor is produced by the body and is able to promote nerve cell survival, growth and differentiation. BDNF is a member of the neurotrophic factor family and serves a key role in maintaining the normal physiological function of neurons. BDNF promotes neurotransmitter release and enhances neuromuscular transmission excitation-contraction coupling. In addition, it regulates ion channel activity through the high-affinity receptor protein, TrkB, and the low-affinity receptor protein, p75 $(17,18)$.

In the present study, according to the autoimmune mechanism of TS, the rat striata were infused with serum from TS patients, which contained a high concentration of ABGA, resulting in striatal dysfunction and various stereotypic behaviors. A number of endogenous neuroprotective responses are known to be generated against noxious stimuli (19), with nutrition regulation being an important neuroprotective mechanism. In addition, noxious stimulation of the brain can cause upregulation of BDNF (20). BDNF stabilizes the metabolic function of damaged neurons, in which synthesis and metabolism at low levels (21), and enhances neuron resilience to hypoxia and survival in a damaged environment. Furthermore, BDNF is involved in the development of striatal neurons, nutrition-associated maintenance and neuroprotection $(22,23)$. Antigen-antibody immune response may lead to compensatory protective 
response in the TS rat brain, which is associated with the increased brain BDNF expression levels.

ABGA is able to destroy cells and cause corresponding neurological deficits. However, it may also increase the expression and secretion of endogenous neuroprotective factors, and ultimately induce the expression of neurotrophic factors, such as BDNF, initiating the self-protection and repair mechanisms of the body (24).

BDNF has been reported to have a significant nutritional effect on dopamine neurons and to increase the number of dopamine receptors in the brain (25). In certain cases, excess BDNF is harmful. Excessive expression of BDNF may damage the neural circuitry, which could affect memory and cognitive function, increasing the risk of seizures (26). Dopamine receptor levels have been shown to increase in the brains of infants with TS (27). The experimental results of the current study showed that BDNF levels were elevated in TS rats. Thus, we hypothesize that BDNF participates in the pathogenesis of TS by increasing the number of dopamine receptors and by upregulating the excitatory ion channels and downregulating the inhibitory ion channels in order to release neurotransmitters.

MSC has immunosuppressive properties in vivo. In an inflammation environment, MSC may induce the immune regulation process, limit inflammation to facilitate self-survival and form an immunosuppressive microenvironment. Furthermore, MSC has anti-inflammatory and immunomodulatory effects, and inhibits the proliferation of T lymphocytes $(28,29)$.

The present results indicate that antigen-antibody reaction may cause immune damage and lead to a series of stereotypic behaviors in autoimmune TS rats. Upon transplantation into the striatum of TS rats, MSCs inhibit the immune response and repair local damage in the microenvironment to restore homeostasis, resulting in the elevated BDNF levels in TS rats returning to the normal levels. In the present study, following MSC transplantation, the BDNF level was reduced in the striatum of TS rats. The excitatory neurotransmitter channels were closed and the inhibiting ion channels were increased, thereby improving neuromotor function and reducing stereotypy in the TS rats. In conclusion, MSC transplantation in TS rats may reduce BDNF levels and reduce the stereotyped behavior. However, the mechanism underlying MSC transplantation for the treatment of TS requires further study.

\section{Acknowledgements}

This study was supported by grants from the National Natural Science Foundation of China (no. 81101017), the Department of Science and Technology of Shandong Province (no. BS2010F030), and the Yantai Science and Technology Development project (no. 2010148-24).

\section{References}

1. Tamara P: Tourette syndrome and other tic disorders of childhood. Handb Clin Neurol 112: 853-856, 2013.

2. Cheung MY, Shahed J and Jankovic J: Malignant tourette syndrome. Mov Disord 22: 1743-1750, 2007.

3. Lv W, Li WY, Xu XY, Jiang H and Bang OY: Bone marrow mesenchymal stem cells transplantation promotes the release of endogenous erythropoietin after ischemic stroke. Neural Regen Res 10: 1265-1270, 2015.
4. Choi HS, Kim HJ, Oh JH,Park HG, Ra JC, Chang KA and Suh YH: Therapeutic potentials of human adipose-derived stem cells on the mouse model of Parkinson's disease. Neurobiol Aging 36: 2885-2892, 2015

5. Liu X, Wang Y, Li D and Ju X: Transplantation of rat neural stem cells reduces stereotypic behaviors in rats after intrastriatal microinfusion of Tourette syndrome sera. Behav Brain Res 186: 84-90, 2008.

6. Liu XM, Wang YW and Yi MJ: Effects of human mesenchymal stem cell transplantation in the bilateral corpus striatum in a rat model of Tourette's syndrome. Neural Regen Res 5: 1285-1290, 2010.

7. van Velthoven CT, Braccioli L, Willemen HL, Kavelaars A and Heijnen CJ: Therapeutic potential of genetically modified mesenchymal stem cells after neonatal hypoxic-ischemic brain damage. Mol Ther 22: 645-654, 2014.

8. Liu X, Wang X, Li L, Wang H and Jiao X: Influence of mesenchymal stem cell transplantation on stereotypic behavior and dopamine levels in rats with Tourette syndrome. PLoS One 8: e62198, 2013.

9. Chen A, Xiong LJ, Tong Y and Mao M: The neuroprotective roles of BDNF in hypoxic ischemic brain injury. Biomed Rep 1: 167-176, 2013.

10. Hornig M and Lipkin WI: Immune-mediated animal models of Tourette syndrome. Neurosci Biobehav Rev 37: 1120-1138, 2013.

11. Singer HS, Loiselle CR, Lee O, Minzer K, Swedo S and Grus FH: Anti-Basal Ganglia Antibodies in PANDAS. Mov Disord 19: 406-415, 2004

12. Macri S, Onori MP, Roessner V and Laviola G: Animal models recapitulating the multifactorial origin of Tourette syndrome. Int Rev Neurobiol 112: 211-237, 2013.

13. Taylor JL, Rajbhandari AK, Berridge KC and Aldridge JW: Dopamine receptor modulation of repetitive grooming actions in the rat: Potential relevance for Tourette syndrome. Brain Res 1322: 92-101, 2010.

14. Malaty IA and Akbar U: Updates in medical and surgical therapies for Tourette syndrome. Curr Neurol Neurosci Rep 14: 458, 2014.

15. Phinney DG and Isakova IA: Mesenchymal stem cells as cellular vectors for pediatric neurological disorders. Brain Res 1573: 92-107, 2014.

16. Zhang F, Wang YY, Liu H, Lu YF, Wu Q, Liu J and Shi JS: Resveratrol produces neurotrophic effects on cultured dopaminergic neurons through prompting astroglial BDNF and GDNF release. Evid Based Complement Alternat Med 2012: 937605, 2012.

17. Kordi-Tamandani DM, Sahranavard R and Torkamanzehi A: DNA methylation and expression profiles of the brain-derived neurotrophic factor (BDNF) and dopamine transporter (DAT1) genes in patients with schizophrenia. Mol Biol Rep 39: 10889-10893, 2012.

18. Ferrini F and De Koninck Y: Microglia control neuronal network excitability via BDNF signalling. Neural Plast 2013: 429815, 2013.

19. Paulsen JS: Cognitive impairment in Huntington disease: Diagnosis and treatment. Curr Neurol Neurosci Rep 11: 474-483, 2011.

20. Shimada H, Makizako H, Doi T, Yoshida D, Tsutsumimoto K, Anan Y, Uemura K, Lee S, Park H and Suzuki T: A large, cross-sectional observational study of serum BDNF, cognitive function and mild cognitive impairment in the elderly. Front Aging Neurosci 6: 69, 2014.

21. Li Y, Yui D, Luikart BW, McKay RM, Li Y, Rubenstein JL and Parada LF: Conditional ablation of brain-derived neurotrophic factor-TrkB signaling impairs striatal neuron development. Proc Natl Acad Sci USA 109: 15491-15496, 2012.

22. Niitsu T, Shirayama Y, Matsuzawa D, Hasegawa T, Kanahara N, Hashimoto T, Shiraishi T, Shiina A, Fukami G, Fujisaki M, et al: Associations of serum brain-derived neurotrophic factor with cognitive impairments and negative symptoms in schizophrenia. Prog Neuropsychopharmacol Biol Psychiatry 35: 1836-1840, 2011.

23. Mang CS, Campbell KL, Ross CJ and Boyd LA: Promoting neuroplasticity for motor rehabilitation after stroke: Considering the effects of aerobic exercise and genetic variation on brain-derived neurotrophic factor. Phys Ther 93: 1707-1716, 2013.

24. Martino D, Chiarotti F, Buttiglione M, Cardona F, Creti R, Nardocci N, Orefici G, Veneselli E and Rizzo R; Italian Tourette Syndrome Study Group: The relationship between group A streptococcal infections and Tourette syndrome: A study on a large service-based cohort. Dev Med Child Neurol 53: 951-957, 2011.

25. Yeh TK, Hu CY, Yeh TC, Lin PJ, Wu CH, Lee PL and Chang CY: Association of polymorphisms in BDNF, MTHFR, and genes involved in the dopaminergic pathway with memory in a healthy Chinese population. Brain Cogn 80: 282-289, 2012. 
26. Binder DK, Croll SD, Gall CM and Scharfman HE: BDNF and epilepsy: Too much of a good thing? Trends Neurosci 24: 47-53, 2001.

27. Denys D, de Vries F, Cath D, Figee M, Vulink N, Veltman DJ, van der Doef TF, Boellaard R, Westenberg H, van Balkom A, et al: Dopaminergic activity in Tourette syndrome and obsessive-compulsive disorder. Eur Neuropsychopharmacol 23: $1423-1431,2013$
28. Stagg J and Galipeau J: Mechanisms of immune modulation by mesenchymal stromal cells and clinical translation. Curr Mol Med 13: 856-867, 2013.

29. Brennen WN, Denmeade SR and Isaacs JT: Mesenchymal stem cells as a vector for the inflammatory prostate microenvironment. Endocr Relat Cancer 20: R269-R290, 2013. 